\title{
Question: A 62-year-old lady with painful swelling of right hand index finger for the last 8 weeks
}

\author{
Sajid Butt ${ }^{1} \cdot$ Roberto Tirabosco ${ }^{2} \cdot$ Asif Saifuddin $^{1}$ \\ Published online: 5 August 2020 \\ (C) ISS 2020
}

\section{Discussion}

Plain radiograph of the right hand shows mild expansion of the proximal phalanx of the index finger, with thickening and irregularity of the cortex and a permeative pattern of bone destruction (Fig. 1). The medial and lateral margins of the proximal phalanx show cortical thickening secondary to organised periosteal reaction. A small fleck of calcification was seen at the medial margin of the cortex of proximal phalanx, but no matrix mineralisation was identified. Soft tissue swelling was present around the phalanx. The diagnosis was felt to be that of a high-grade chondrosarcoma. MRI (Fig. 2) demonstrated intermediate T1-weighted and slightly increased STIR signal intensity within the phalanx, with expansion of the medullary cavity and scalloping of the endosteal cortex. Surrounding soft tissue thickening measuring $1.5 \mathrm{~cm}$ was seen along the full length of the phalanx, which also appeared hyperintense on fat-suppressed PDW sequences. A small amount of fluid was seen within the long flexor tendon sheath. Ultrasound examination (Fig. 3) showed that the soft tissue had low echogenicity with increased vascularity at its margins. The lesion looked solid to semi-solid in consistency.

Differential diagnosis of an expansile phalangeal medullary abnormality with chronic cortical thickening and surrounding soft tissue mass would include a chondral lesion (possibly with sarcomatous change), a metastatic deposit or infection (TB, bacterial or fungal). Connective tissue disorders

The case presentation can be found at https://doi.org/10.1007/s00256020-03552-3

Sajid Butt

Sajid.Butt@nhs.net

1 Department of Radiology, Royal National Orthopaedic Hospital, Stanmore, UK

2 Department of Histopathology, Royal National Orthopaedic Hospital, Stanmore, UK like sarcoidosis can also be considered in the differential diagnosis.

An ultrasound-guided needle biopsy was performed, and histopathology demonstrated caseating granulomata with multiple Langhans giant cells (one such cell has been annotated with arrows in Fig. 4 reproduced in the answer section). A diagnosis of tuberculous infection was made and anti-TB chemotherapy started, making a full recovery after completing a year-long treatment.

The incidence of musculoskeletal TB is around 10\% [1-4]. The incidence of TB dactylitis is $1 \%$ being seen more frequently in children [5-7]. The disease typically has an indolent onset and can present with swelling, pain and low-grade fever $[7,8]$.

Expansion of the phalanx has historically been described as 'spina ventosa' [5]. This term signifies the presence of cystic expansion of the short tubular bones of the hands or feet. In our case, along with medullary expansion, the presence of a large painful soft tissue mass was a prominent clinical feature. In reported cases of TB dactylitis, soft tissue masses are seen but are usually painless and can be accompanied by draining sinuses [7-10]. The radiographic findings and the MR images in our case showed bone expansion and a large soft tissue mass, felt to be consistent with a primary bone tumour such as high-grade chondrosarcoma. However, the presence of flexor tenosynovitis indicated a possible infective aetiology, and histopathology confirmed the diagnosis of granulomatous infection.

Tuberculous dactylitis can be a delayed diagnosis even in children, and particularly when occurring in an atypical age as in this case [10]. A careful observation of ancillary signs of possible infection such as osteolysis, chronic periosteal thickening and fluid in the tendon sheath and surrounding soft tissues should alert the radiologists to a possible infective aetiology. The biopsy specimen should therefore be sent for both histopathology and for appropriate microbiology.

The condition is treated with multi-drug chemotherapy comprising 3-4 drugs which is required for a period of 9 
12 months [4]. Surgery performed for the purpose of curettage and or drainage of abscess has a role in a relatively smaller number of patients [10].

\section{References}

1. Peto HM, Pratt RH, Harrington TA, LoBue PA, Armstrong LR. Epidemiology of extrapulmonary tuberculosis in the United States, 1993-2006. Clin Infect Dis. 2009;49(9):1350-7.

2. Mazza-Stalder J, Nicod L, Janssens JP. Extrapulmonary tuberculosis. Rev Mal Respir. 2012;29(4):566-78.

3. Watts HG, Lifeso RM. Tuberculosis of bones and joints. J Bone Joint Surg Am. 1996;78(2):288-98.

4. World Health Organisation. Global Tuberculosis Report 2019 [Available from: https://apps.who.int/iris/bitstream/handle/10665/ 329368/9789241565714-eng.pdf?ua=1.
5. Sahli H, Roueched L, Sbai MA, Bachali A, Tekaya R. The epidemiology of tuberculous dactylitis: a case report and review of literature. Int J Mycobacteriol. 2017;6(4):333-5.

6. John BM, Muthuvel S, Gupta S. Multicentric tubercular dactylitis. Med J Armed Forces India. 2007;63(2):186-7.

7. Thatoi P, Parida M, Barik R, Das B. Multifocal tubercular dactylitis: a rare presentation of skeletal tuberculosis in an adult. $\mathrm{J}$ Clin Diagn Res. 2017;11(6):Od23-od4.

8. Sbai MA, Benzarti S, Sahli H, Sbei F, Maalla R. Osteoarticular tuberculosis dactylitis: four cases. Int J Mycobacteriol. 2015;4(3): $250-4$.

9. Rao GN, Gali JH, Rao SN. Tuberculous dactylitis: an uncommon presentation of a common infection. Case reports in pediatrics. 2016;2016:4013471.

10. Ritz N, Connell TG, Tebruegge M, Johnstone BR, Curtis N. Tuberculous dactylitis-an easily missed diagnosis. Eur J Clin Microbiol Infect Dis. 2011;30(11):1303-10.

Publisher's note Springer Nature remains neutral with regard to jurisdictional claims in published maps and institutional affiliations. 